integral part of the neurologic examination.

But all this changed as neurology and neuropathology expanded. It was then realized that there was not one but many diseases that could express themselves as a progressive mental decline, some treatable and even curable. In practice, the definition of these clinical syndromes answered to the criteria of chronicity, progressivity and a symptomatology the essence of which was intellectual deterioration. The main problem, as Critchley pointed out in 1938, was the definition of intellectual functions.

Following Spearman and other psychologists, intelligence was found to be multifactoral, comprised minimally of memory, language, reasoning, calculation and visualspatial orientation. Clinicians learned that each of these functions had its own anatomy and could be lost singly or in various combinations during the course of disease. Moreover, it was ascertained that certain patterns of deficit might be linked to specific diseases, e.g. pure global memory deficit to vitamin deficiency. Therefore, it is a mark of laxity in thinking to use the term dementia in a generic sense; always there should be a qualifying adjective, e.g. global dementia, amnesic dementia, dysphasic dementia, etc.

This is a criticism that could be levelled at this monograph - that it tends to lump together all clinical states in which there is some alteration or impairment of mental function. Only the acute confusional psychoses and deliria are separated. While a common practice in psychiatry, for clinical neurologists this is a step backwards.

From clinical experience most neurologists have come to think of the dementing diseases as being chronic and progressive. Only a relatively small number satisfy these criteria. Yet the subject matter of this monograph includes more than a hundred diseases. The reason for this extreme inclusiveness is that the temporal factor in pathology is disregarded. No importance is attached to the mode of onset, the clinical course and outcome as denominating attributes of disease. To include every disease that touches the mind in any way as a dementia, regardless of its temporal profile, as is inferred in this monograph, serves no useful purpose.

Whitehouse is aware of these problems and struggles with matters of definition. The standard criteria of the American Psychiatric Association's Mental and Statistical Manual (DSM III R) are quoted but many neurologists find them inadequate, and the same may be said of the formulations of the special committee on dementia of the National Institute of Neurologic Diseases and Stroke.

Despite the reviewers' criticism of the wide compass of subject material, the main topic of this monograph is Alzheimer's and closely related diseases, and the various contributors have covered the subject well. The clinical descriptions are of good quality and the first six chapters on epidemiology, genetics, neuropathology and neuropsychology are informative and up-to-date. Helpful to the readers are accounts not only of the most recent scientific data but also of the methodology by which they were obtained. Even in the later descriptions of vascular, neoplastic, toxic, infectious and demyelinative diseases, which may cause derangements of mind, it is their psychologic aspects which are stressed. This section finishes with a critical assessment of therapies, both for the disease process and some of the unwanted symptoms it produces.

A final section contains comments on the psychologic and social implications of a dementing disease. The role of the physician in helping the family provide humane care is presented well. Wise suggestions are to be found in these pages.

In general, the book is strongly recommended. It should attract a wide readership drawn from the fields of neurology, psychiatry and the neurosciences. It measures up to the standards set by previous editions of Davis' Contemporary Neurology series.

RD ADAMS

Stereotactic and Image-Directed Surgery of Brain Tumours. Edited by DAvid G T THOMAS. (Pp 236 Illustrated; Price: $£ 70.00)$. 1993. Edinburgh, Churchill Livingstone. ISBN 0-443-04445-7.

Currently there is a very strong trend towards minimally interventive forms of surgery. Far from standing aloof from such a movement neurosurgeons can be counted among its earliest pioneers. Since the introduction of stereotactic frames for approaching indirectly remote intracranial targets in humans in 1947 by Spiegel and Wycis in North America and in 1949 by Leksell in Europe there has been a steady improvement in increasingly flexible and precise methods for accessing the brain. Thi progress has been much aided by the dramatic development of new imaging techniques. Despite the increasingly rapid rate of change in scientific progress this book encapsulates the currently available advances in brain tumour surgery, emphasising the importance and pitfalls of stereotactic biopsy, CT and MR guided surgery, as well as the role of minimally interventive interstitial radiotherapy and completely non-interventive radiosurgery, the latter both by gamma knife and by modified linear accelerator. However, perhaps the most fascinating contributions are those dealing with the mathematical principles necessary for the pursuit of better three dimensional imaging, and with the awesome prospect in the near future of how much of today's activity will soon be mastered by robots. The promise of holographic imaging frameless stereotaxy, selective radiosensitisers and robots manipulating increasingly sophisticated endoscopes is exciting indeed. DMC FORSTER

Carpal Tunnel Syndrome and Other Disorders of the Median Nerve. By RICHARD ROSENBAUM and JOSE OCHOA.

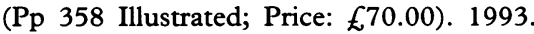
Oxford, Butterworth-Heinemann. ISBN 07506-9229-4.

The carpal tunnel syndrome is one of the commoner neurological conditions seen by a broad spectrum of medical and surgical practitioners. The appearance of a monograph devoted to it is timely in view of its recent designation as a prescribed industria disease in the UK. While the principal aim of the authors has been to describe this and other lesions of the median nerve, they have used it as an "archetype for other focal compressive neuropathies" and in doing so have incorporated much of their vast experience in the field of pathophysiology of chronic nerve compression.

The main sections of the book contain an excellent chapter on the anatomy of the median nerve and cover the clinical aspects, including presentation, diagnostic criteria and differential diagnosis, aetiological mechanisms and its association with other medical conditions. There is a detailed and critical review of the electrophysiological and other methods employed for diagnostic evaluation and of the conservative and surgical management of the condition. They also discuss the relationship between activity and occupations which may be casually related to carpal tunnel syndrome and associated medico-legal issues. There is a brief but challenging review of the controversial issues of "causalgia" and "reflex sympathetic dystrophy" related to median nerve injuries. The final chapters describe the various proximal median nerve compression syndromes and digital nerve lesions. The book is well illustrated, with excellent anatomical line drawings and instructive patient photographs in the clinical sections.

This is an excellent monograph on the subject and a lot more besides. The authors are to congratulated for combining their considerable experience of pathophysiology of chronic nerve compression with information gleaned from "well over 2,000 articles and books" on the subject. Every one who deals with the clinical, diagnostic or surgical aspects of median or other nerve disorders should read it.

PRW FAWCETT

\section{SHORT NOTICE}

Yearbook of Neurology and Neurosurgery 1993. A Mosby Year Book. Edited by WALTER G BRADLEY and ROBT $M$ CROWELL. (Pp 464; Price: $£ 52.00) .1993$. London, Mosby-Year Book Europe Ltd. ISBN 0-8151-2142-3.

The excellent Year Book continues with Walter Bradley as its new Neurology editor. $\mathrm{He}$ has changed the format, introducing three succinct topical review papers. He has co-opted 12 Associate editors to select and provide the brief comments after each paper abstracted. New headings to break up each paper are a new feature: Background, Methods, Findings and Conclusions.

A valuable source of information and further references which is digestible and pleasant to read. 